\title{
Synthesis and Characterization of a Size Series of Extremely Small Thiol-Stabilized CdSe Nanocrystals
}

\author{
Andrey L. Rogach,,$\pitchfork$ Andreas Kornowski, ${ }^{\dagger}$ Mingyuan Gao, ${ }^{\S}$ Alexander Eychmiiller,,$\stackrel{ }{*}$ and \\ Horst Weller ${ }^{\dagger}$ \\ Institut für Physikalische Chemie, Universität Hamburg, 20146 Hamburg, Germany, and \\ Max-Planck-Institut für Kolloid- und Grenzflächenforschung, 12489 Berlin, Germany \\ Received: December 31, 1998; In Final Form: March 11, 1999
}

\begin{abstract}
As an expansion to the wet chemical route for the preparation of quantum-sized II-VI semiconductor materials, a series of thiol-capped crystalline CdSe nanoparticles has been synthesized in aqueous solution using mercaptoalcohols (2-mercaptoethanol, 1-thioglycerol), and mercapto acids (thioglycolic acid, thiolactic acid) as stabilizers. The smaller (app. 1.4-2.2 nm diameter) CdSe particles were obtained using thioalcohols as capping agents; the use of thioacids as stabilizers produced larger (2.1-3.2 nm diameter) CdSe particles. CdSe nanoparticles were separated from the crude solutions as redissolvable powder samples with narrow size distributions using a size-selective fractionation and have been characterized by $\mathrm{UV}$-vis absorption and photoluminescence spectroscopy, X-ray diffraction, high-resolution transmission electron microscopy, and energy-dispersive X-ray analysis. A calculation of the HOMO-LUMO gap of CdSe particles as a function of their size has been done using an extended effective mass approximation.
\end{abstract}

\section{Introduction}

A number of exciting size- and surface-dependent properties of nanometer-sized semiconductor particles, ${ }^{1}$ which lie between molecular and bulk forms of matter, have stimulated an exponential development of nanochemistry and nanophysics in the past decade which is currently being realized in nanotechnology. ${ }^{2}$ Extensive research has been done in the synthesis and characterization of colloidal II-VI semiconductor nanoparticles. An important landmark in the development of wet chemical routes for cadmium chalcogenide nanocrystals was, together with the nonaqueous TOP/TOPO (trioctyl phosphine/trioctyl phosphine oxide) technique, ${ }^{3}$ the use of different thiols as stabilizing agents in aqueous solution. ${ }^{4-6}$ A monodisperse size series of thioalcohol-stabilized $\mathrm{CdS}^{7}$ and $\mathrm{CdTe}^{8}$ nanoparticles with extremely small sizes $(1-3 \mathrm{~nm}$ size range) was synthesized in aqueous solutions and was obtained in the gram scale as redissolvable nanocrystalline powders or as crystals of nanoclusters (superstructures). 9,10 To complete the series of thiolstabilized cadmium chalcogenide nanocrystals synthesized by a wet chemical route in aqueous solution, CdSe nanoparticles were synthesized. The basic spectroscopic and structural characterizations of thiol-capped CdSe nanoparticles are presented, reserving a more detailed study of some selected CdSe samples for future investigations.

\section{Experiment}

Chemicals. All chemicals used were of analytical grade or of the highest purity available. They were obtained from Sigma, Merck, Aldrich, Alfa, and Fluka and used as received. The

* Corresponding author. E-mail: eychmuel@chemie.uni-hamburg.de. Fax: +49-40 428383452.

$\dagger$ Universität Hamburg

$\doteqdot$ Permanent address: Physico-Chemical Research Institute, Belarussian State University, 220050 Minsk, Belarus.

$\S$ Max-Planck-Institut. solution of $0.05 \mathrm{M}$ NaHSe was prepared following the synthesis route of NaHTe solution described previously. ${ }^{8}$ Under a $\mathrm{N}_{2}$ atmosphere, $100 \mathrm{~mL}$ of $0.05 \mathrm{M} \mathrm{NaOH}$ solution was titrated with $\mathrm{H}_{2} \mathrm{Se}$ (generated by the reaction of $\mathrm{Al}_{2} \mathrm{Se}_{3}$ with $10 \% \mathrm{H}_{2} \mathrm{SO}_{4}$ ). $\mathrm{Al}_{2} \mathrm{Se}_{3}$ was used in a 1.7-fold excess to the calculated quantity. After titration, the solution was bubbled with $\mathrm{N}_{2}$ for $30 \mathrm{~min}$ to remove any traces of $\mathrm{H}_{2} \mathrm{Se}$ present in the solution. The solution of NaHSe can be stored under nitrogen for some days but in this case was used directly after preparation.

Apparatus. Room-temperature UV-vis absorption spectra were obtained with a Perkin-Elmer Lambda 40 UV-vis spectrophotometer. Photoluminescence measurements were performed at room temperature using a FluoroMax-2 spectrofluorimeter (Instruments SA). X-ray powder diffraction (XRD) spectra were taken on a Philips X'Pert diffractometer $(\mathrm{Cu} \mathrm{K} \alpha$ radiation, variable entrance slit, Bragg-Brentano geometry, secondary monochromator). Samples for these measurements were prepared by placing finely dispersed powders of $\mathrm{CdSe}$ nanoparticles on standard PVC supports. High-resolution transmission electron microscopy (HRTEM) and energy-dispersive $\mathrm{X}$-ray analysis (EDX) were performed on a Phillips CM-300 microscope operating at $300 \mathrm{kV}$. TEM samples were prepared by dropping diluted aqueous solutions of $\mathrm{CdSe}$ nanoparticles onto 400-mesh carbon-coated copper grids with the excess solvent immediately evaporated.

\section{Results and Discussion}

Synthesis of CdSe Nanoparticles. Aqueous colloidal solutions of CdSe nanoparticles have been synthesized through the addition of freshly prepared oxygen-free NaHSe solutions to $\mathrm{N}_{2}$-saturated $\mathrm{Cd}\left(\mathrm{ClO}_{4}\right)_{2} \cdot 6 \mathrm{H}_{2} \mathrm{O}$ solutions at $\mathrm{pH} 11.2$ in the presence of different thiols as stabilizing agents. The CdSe particle size was controlled by the type of stabilizer and through postpreparative size-selective precipitation. As has been shown in our previous studies, ${ }^{7,8}$ thioalcohols (2-mercaptoethanol and 1-thioglycerol) are effective size-regulating and stabilizing 

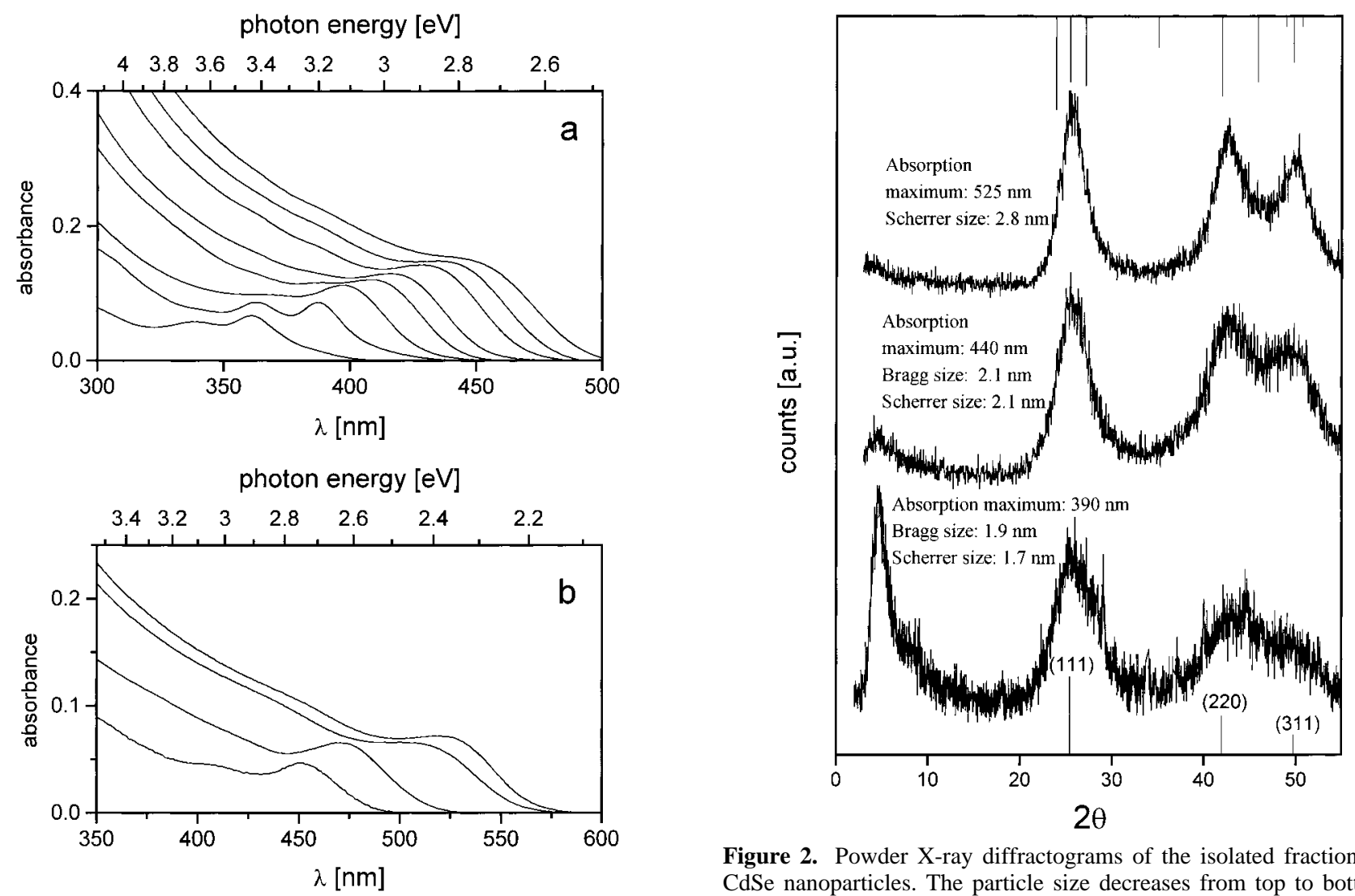

Figure 1. Absorption spectra of the isolated fractions of CdSe nanoparticles capped with 2-mercaptoethanol (a) and thioglycolic acid (b).

agents for cadmium chalcogenide nanoparticles. In addition, thioglycolic and thiolactic acids were also used as stabilizers for CdSe nanoparticles as reported by Gao et al. ${ }^{11}$

A solution of $1.97 \mathrm{~g}(4.70 \mathrm{mmol})$ of $\mathrm{Cd}\left(\mathrm{ClO}_{4}\right)_{2} \cdot 6 \mathrm{H}_{2} \mathrm{O}$ and $11.54 \mathrm{mmol}$ of the stabilizer (RSH) in $250 \mathrm{~mL}$ of demineralized water was adjusted to $\mathrm{pH} 11.2$ with $1 \mathrm{M} \mathrm{NaOH}$. The solution was placed in a three-necked flask fitted with a septum and valves and was deaerated with $\mathrm{N}_{2}$ bubbling for 30 min. Under vigorous stirring, $44 \mathrm{~mL}(2.2 \mathrm{mmol})$ of the freshly prepared oxygen-free $0.05 \mathrm{M}$ NaHSe solution was injected. The initial molar ratio $\mathrm{Cd}^{2+} / \mathrm{Se}^{2-} / \mathrm{RSH}$ was therefore app. 1:0.5:2.4, which were the same conditions used for the preparation of thiolstabilized $\mathrm{CdS}^{7}$ and $\mathrm{CdTe}^{8}$ nanoclusters.

The addition of NaHSe produced bright-yellow, transparent colloids stable toward oxidation under air. Solutions were refluxed for different times (up to $12 \mathrm{~h}$ ), and aliquots were removed at regular intervals $(15-30 \mathrm{~min})$ with absorption spectra taken to monitor the growth of the clusters. The development of absorption spectra of thioalcohol-stabilized CdSe nanoparticles with time resembles that of thioalcohol-stabilized $\mathrm{CdS}$ and CdTe nanoparticles. ${ }^{7,8}$ Addition of the NaHSe solution caused the appearance of absorption shoulders or maxima in the UV region and unstructured absorption features at longer wavelengths. The heating of the solutions led to an increase of absorption between 330 and $400 \mathrm{~nm}$ at the expense of the shortwavelength bands. New absorption maxima appeared at specific wavelengths, suggesting the formation of some thermodynamically favorable cluster structures. A prolonged refluxing (up to $12 \mathrm{~h}$ ) caused a continuous red shift of the absorption edge up to $450 \mathrm{~nm}$ which was not accompanied with a formation of new pronounced absorption maxima. Particle growth occurred continuously at this stage via Ostwald ripening.

Figure 2. Powder X-ray diffractograms of the isolated fractions of CdSe nanoparticles. The particle size decreases from top to bottom; particle sizes obtained from Bragg and Scherrer equations are shown. The line spectra give the bulk CdSe reflections (top, wurzite hexagonal; bottom, zinc blende cubic).

In contrast, no or only very weakly pronounced absorption maxima were observed at the initial stage of the synthesis of $\mathrm{CdSe}$ nanoparticles in the presence of thioacids, and the absorption edge which appeared after addition of NaHSe solution lay in the visible spectral range. During the heating of the solution, the particle growth proceeded about 5 times faster than for thioalcohol-stabilized CdSe and larger CdSe particles were formed. A prolonged refluxing (up to $12 \mathrm{~h}$ ) of the solutions of thioacid-stabilized CdSe nanoparticles caused a red shift of the absorption edge up to $530-540 \mathrm{~nm}$ due to continuous growth of the particles.

Isolation of CdSe Nanoparticle Fractions: Absorption and Photoluminescence of the Particles. Absorption spectra of the crude colloidal solutions represented a mixture of CdSe nanoparticles with different sizes. The method of size-selective precipitation $^{12}$ was used to isolate the samples of CdSe nanoparticles with different sizes and narrow size distributions from crude solutions. They were concentrated down to app. 30 $\mathrm{mL}$ using a rotary evaporator, and 2-propanol was added dropwise until turbidity occurred. The solutions were stirred for $2-3 \mathrm{~h}$, and the precipitate and supernatant were separated through centrifugation.

Figure 1 shows sets of absorption spectra of mercaptoethanol and thioglycolic acid stabilized CdSe nanoparticles with different sizes separated from the corresponding crude solutions. The narrowing of the size distribution of the particles led to a better resolution of the $1 \mathrm{~s}-1 \mathrm{~s}$ electronic transitions. As expected for quantum-sized particles, the position of the first electronic transition was shifted to higher photon energies and became more pronounced with decreasing particle size in accordance with the size quantization effect. ${ }^{1}$ Higher electronic transitions were also clearly resolved for the smallest CdSe nanoparticles (Figure 1). 


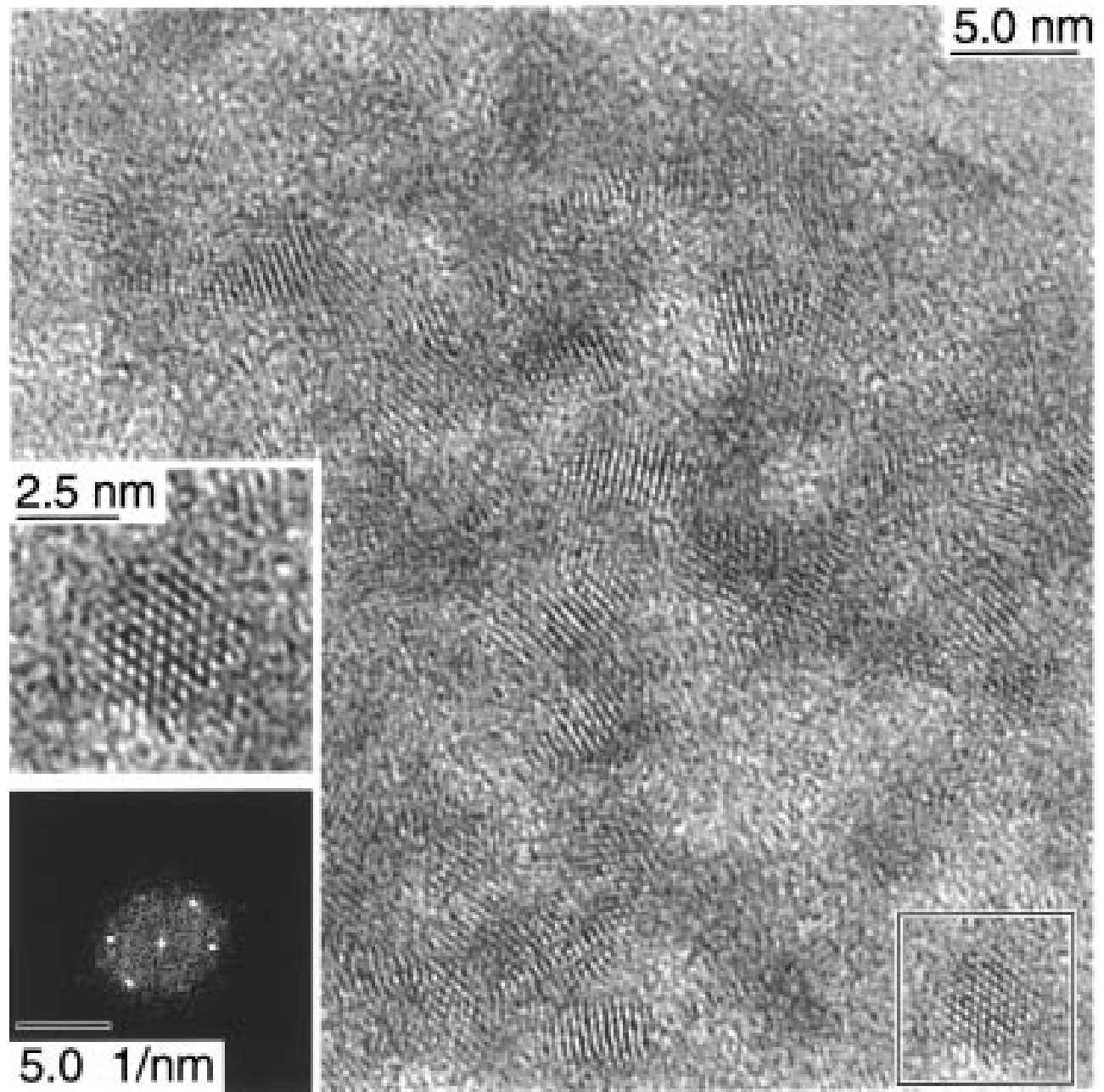

Figure 3. HRTEM overview image of thioglycerol-stabilized CdSe nanoparticles together with a single CdSe particle and its corresponding FFT.

Both thioalcohol- and thioacid-stabilized CdSe nanoparticles showed a broad photoluminescence band with a room temperature photoluminescence quantum yield of less than $0.1 \%$ (referenced by Rhodamin 6G) and a maximum which shifted strongly toward longer wavelengths in comparison with the absorption edge. This kind of photoluminescence which has been ascribed to an emission from deep surface traps was also observed for a series of thioalcohol-capped CdS clusters. ${ }^{13}$ The photoluminescence maxima of thioalcohol-stabilized $\mathrm{CdSe}$ particles were located at about $600 \mathrm{~nm}$ and were further shifted toward the near IR for thioacid-stabilized ones.

Structural Characterization of CdSe Nanoparticles. Smalland wide-angle powder X-ray diffractometry has been performed on the powders of isolated fractions of $\mathrm{CdSe}$ nanoparticles. Figure 2 shows X-ray diffraction patterns of some selected fractions of CdSe nanoparticles capped with different stabilizers. The diffractograms confirmed the crystallinity of the CdSe nanoparticles. The positions of the reflection peaks matched the cubic modification of CdSe (zinc blende phase) better than the hexagonal one. Note that the cubic modification has also been found to be more preferable for thiol-capped $\mathrm{CdS}$ and $\mathrm{CdTe}$ nanocrystals 7,8 synthesized in aqueous solution at moderate temperatures as opposed to the hexagonal phase for cadmium chalcogenides obtained through the high-temperature TOP/ TOPO technique. ${ }^{3}$ Alkoxysilane-stabilized CdSe nanocrystals exhibited cubic zinc blende structure. ${ }^{14}$ Modeling of the experimentally observed XRD spectra using the hexagonal modification of CdSe with the presence of stacking faults along the (002) axis has been performed by Murray et al., ${ }^{3}$ possibly fitting our data for the smaller CdSe nanoparticles better than the cubic modification. An extensive structural characterization of thiol-stabilized CdSe nanoparticles will be left for future investigations.

The broadness of the diffraction peaks of CdSe nanoparticles shown in Figure 2 increased gradually with a decrease of particle size. The mean nanocrystal sizes obtained from the full width at half-maximum intensity of the (111) zinc blende reflection according to the Scherrer equation are depicted in Figure 2. A reflection maximum appeared also in the small-angle region of the X-ray diffraction patterns due to the periodicity of their arrangement. The appearance of such a peak provides further confirmation for the narrow size distribution of the particles. The peak angle maxima can be converted to the nearest neighbor distances of the nanoparticles in the powdered samples by using the Bragg equation. These distances can be used as a measure of the mean particle size as has been discussed in refs 7 and 8 . The calculated values are depicted in Figure 2 and are in surprisingly good agreement with the ones obtained from the Scherrer equation.

Figure 3 shows a typical HRTEM overview image of CdSe nanoparticles (here thioglycerol-stabilized CdSe particles with the excitonic maximum in the absorption spectrum at ca. 375 $\mathrm{nm})$ together with a single $\mathrm{CdSe}$ particle and its corresponding fast Fourier transform (FFT). The existence of lattice planes on the HRTEM image further confirmed the crystallinity of CdSe nanoparticles. The average sizes estimated from HRTEM micrographs were generally larger than those obtained from XRD patterns, a trend already observed for thiol-stabilized CdS 
TABLE 1: Comparison of Size, Electronic Transition Energies, Crystal Structure, and Photoluminescence Behavior of CdSe Nanoparticles Capped with Different Stabilizers

\begin{tabular}{lll}
\hline stabilizer & 2-mercaptoethanol & 1-thioglycerol \\
\hline crystal structure & cubic CdSe (zinc blende) & cubic CdSe (zinc blende) \\
photoluminescence & trapped & trapped \\
1s -1 s electronic transition, $n m$ & $360-450$ & $360-450$ \\
diameter, nm & $1.4-2.2$ & $1.4-2.2$
\end{tabular}



Figure 4. Calculated energy of the first electronic transition as a function of the CdSe nanoparticle size together with experimental values for some fractions of CdSe nanoparticles. Two regions of the particle sizes are depicted for two types of stabilizers.

clusters with comparable sizes. ${ }^{7}$ We note here that electron microscopy in this regime of extremely small sizes may lead to an overestimation of the contribution of larger particles of the given size distribution. Additionally, Rietveld analysis of the XRD spectra reveals larger particle sizes than those obtained through Braggs and Scherrers equations ${ }^{15}$ and thus converging to the particle sizes estimated from the TEM images.

EDX measurements on CdSe nanocrystals indicated the presence of $\mathrm{Cd}, \mathrm{Se}$, and $\mathrm{S}$ with the atomic ratio of $\mathrm{S} / \mathrm{Se}$ increasing with decreasing of the particle sizes. This correlates with the increase of the surface-to-volume ratio when particles become smaller, which corresponds to a larger amount of stabilizing thiol molecules (and S atoms) on the particle surface relative to the number of $\mathrm{Se}$ atoms in the particle core.

Calculation of the 1s-1s Electronic Transition of CdSe Nanoparticles. Calculation of the HOMO-LUMO gap of CdSe nanoparticles as a function of their size (treated as spheres) has been done using an extended theoretical approach described in detail in ref 16. The extension of the common effective mass approximation included the implementation of the Coulomb interaction and finite potential walls at the particle boundaries in water as the surrounding dielectric medium. The physical parameters put into the model were $E_{\mathrm{g}, \mathrm{CdSe}}=1.7 \mathrm{eV},{ }^{17}$ the effective masses $m_{\mathrm{e}, \mathrm{H}_{2} \mathrm{O}}=m_{\mathrm{h}, \mathrm{H}_{2} \mathrm{O}}=1, m_{\mathrm{e}, \mathrm{CdSe}}=0.11, m_{\mathrm{h}, \mathrm{CdSe}}$ $=0.44$, and the high-frequency dielectric constants $\epsilon_{\mathrm{H}_{2} \mathrm{O}}=1.78$, $\epsilon_{\mathrm{CdSe}}=5.8 .^{18}$

The results of the calculation of the $1 \mathrm{~s}-1$ s transition energy in CdSe nanoparticles as a function of particle size are shown in Figure 4 together with experimental values derived from XRD patterns for some fractions of CdSe nanoparticles. Two regions for HOMO-LUMO transition energies were obtained from the absorption spectra of CdSe particles and, accordingly, two size regions are depicted on Figure 4; CdSe nanoparticle sizes from 1.4 to app. $2.2 \mathrm{~nm}$ were achieved using thioalcohols and from app. 2.1 to $3.2 \mathrm{~nm}$ using thioacids as stabilizing agents. CdSe particle sizes obtained from the theoretical curve in Figure 4 are in reasonable agreement with the experimentally determined values.

\section{Summary}

Some characteristics of the series of thiol-stabilized CdSe nanoparticles with different sizes are summarized in Table 1. All particles were in the size quantization regime in comparison with bulk CdSe. Second electronic transitions were clearly resolved for the smallest CdSe nanoparticles. The size-dependent shift of the CdSe particle band gap energies calculated by a finite depth potential well model in the framework of the effective mass approximation was in a reasonable agreement with the experimentally observed change in the absorption spectra of CdSe nanoparticles with size.

CdSe nanoparticles were crystalline and preferentially adopted the cubic zinc blende CdSe phase. Although not favorable for bulk $\mathrm{CdSe},{ }^{19}$ the zinc blende phase appears to be observed for thiol-capped $\mathrm{CdS}, \mathrm{CdSe}$, and CdTe nanoclusters synthesized in aqueous solution at moderate temperature. Photoluminescence of CdSe nanoparticles originated from deep surface traps and was practically independent of particle size.

A detailed structural study of the series of CdSe nanoparticles presented here, in particular a confirmation of the structure of the smallest CdSe clusters with well-defined absorption features, is the aim of future investigations.

Acknowledgment. We thank J. Ludwig from the Mineralogisch-Petrographisches Institut, Universität Hamburg for XRD measurements, and Dr. M. Harrison for carefully reading the manuscript. Financial support was provided by the Volkswagen Foundation, Hannover. M.G. was supported by the Alexander von Humboldt Foundation.

\section{References and Notes}

(1) For reviews, see, e.g.: Brus, L. E. Appl. Phys. A. 1991, 53, 465 Wang, Y.; Herron, N. J. Phys. Chem. 1991, 95, 525. Banyai, L.; Koch, S W. Semiconductor Quantum Dots; World Scientific: Singapore, 1993. Weller, H. Angew. Chem., Int. Ed. Engl. 1993, 32, 41. Weller, H. Adv. Mater. 1993, 5, 88. Alivisatos, A. P. J. Phys. Chem. 1996, 100, 13226. Woggon, U. Optical Properties of Semiconductor Quantum Dots. SpringerVerlag: Berlin, 1997. Gaponenko, S. V. Optical Properties of Semiconductor Nanocrystals. Cambridge University Press: Cambridge, 1998.

(2) See, e.g.: Klein, D. L.; Roth, R.; Lim, A. K. L.; Alivisatos, A. P.; McEuen, P. L. Nature 1997, 389, 699. Feldheim, D. L.; Keating, C. D. Chem. Soc. Rev. 1998, 28, 1. Colvin, V. L.; Schlamp, M. C.; Alivisatos, A. P. Nature 1994, 370, 354. Dabbousi, B. O.; Bawendi, M. G.; Onitsuka, O.; Rubner, M. F. Appl. Phys. Lett. 1995, 66, 1316.

(3) Murray, C. B.; Norris, D. J.; Bawendi, M. G. J. Am. Chem. Soc 1993, 115,8706 .

(4) Rajh, T.; Micic, O. I.; Nozik, A. J. J. Phys. Chem. 1993, 97, 11999.

(5) Lee, G. S. H.; Craig, D. C.; Ma, I.; Scudder, M. L.; Bailey, T. D.; Dance, I. G. J. Am. Chem. Soc. 1988, 110, 4863.

(6) Herron, N.; Calabrese, J. C.; Farneth, W. E.; Wang, Y. Science 1993, 259, 1426.

(7) Vossmeyer, T.; Katsikas, L.; Giersig, M.; Popovic, I. G.; Diesner, K.; Chemseddine, A.; Eychmüller, A.; Weller, H. J. Phys. Chem. 1994, 98, 7665 .

(8) Rogach, A. L.; Katsikas, L.; Kornowski, A.; Su, D.; Eychmüller, A.; Weller, H. Ber. Bunsen-Ges. Phys. Chem. 1996, 100, 1772; 1997, 101, 1668 .

(9) Vossmeyer, T.; Reck, G.; Katsikas, L.; Haupt, E. T. K.; Schulz B.; Weller, H. Science 1995, 267, 1476.

(10) Vossmeyer, T.; Reck, G.; Schulz, B.; Katsikas, L.; Weller, H. J. Am. Chem. Soc. 1995, 117, 12881. 
(11) Gao, M.; Richter, B.; Kirstein, S. Adv. Mater. 1997, 9, 802.

(12) Chemseddine, A.; Weller, H. Ber. Bunsen-Ges. Phys. Chem. 1993, 97, 636 .

(13) Vossmeyer, T. Dissertation. TU Berlin, 1995

(14) Ptatschek, V.; Schreder, B.; Herz, K.; Hilbert, U.; Ossau, W.; Schottner, G.; Rahäuser, O.; Bischof, T.; Lermann, G.; Materny, A.; Kiefer W.; Bacher, G.; Forchel, A.; Su, D.; Giersig, M.; Müller, G.; Spanhel, L. J. Phys. Chem. B 1997, 101, 8898.

(15) Rockenberger, J. Dissertation. University of Hamburg, 1998.
(16) Schooss, D.; Mews, A.; Eychmüller, A.; Weller, H. Phys. Rev. B 1994, 49, 17072

(17) The value $E_{\mathrm{g}, \mathrm{CdSe}}=1.7 \mathrm{eV}$ was obtained by extrapolation of the value $E_{\mathrm{g}, \mathrm{CdSe}}=1.9 \mathrm{eV}$ (cubic CdSe, $4 \mathrm{~K}$, ref 18) to room temperature.

(18) Landolt-Börnstein, Numerical Data and Functional Relationship in Science and Technology, New Series; Springer-Verlag: Berlin, 1982; Group III, Vol. 17, Part b, Section 3.11.

(19) Sidgwick, N. V., Ed. The Chemical Elements and their Compounds; Oxford University Press: London, 1952; Vol. 1, p 271. 